\title{
LA DOMESTICACIÓN DE LA POLÍTICA EXTERIOR DE VENEZUELA (2013-2017)
}

\section{THE DOMESTICATION OF VENEZUELA'S FOREIGN POLICY (2013-2017)}

\author{
Luis Maximiliano Barreto ${ }^{1}$ \\ ORCID: 0000-0002-2932-0046
}

\section{RESUMEN}

El objetivo de este artículo es estudiar la política exterior de Venezuela desde el inicio de la presidencia de Nicolás Maduro hasta la actualidad (2013-2017). Debido al acotado período temporal en consideración y su proximidad con el presente, el trabajo está guiado por una hipótesis preliminar que sostiene que dicha política sufrió un proceso de "domesticación", esto es, una excesiva absorción de la crisis interna en el comportamiento externo del Estado. Al repasar los principales hechos de las relaciones entre Venezuela y sus interlocutores, las acciones se observan continuamente permeadas por la conflictividad doméstica. Esquemáticamente, se pasará revista de los lineamientos previstos en el programa de gobierno y, luego, se abordarán las vinculaciones con los principales actores. A la par de evaluar la validez de la hipótesis preliminar, repasar la propuesta de política exterior permitirá valorar si el accionar externo venezolano se ha mantenido cerca o lejos de ella.

Palabras clave: Política exterior; proceso de domesticación; crisis; conflicto; Venezuela; Nicolás Maduro

\begin{abstract}
The aim of this paper is to study the foreign policy of Venezuela since the beginning of the presidency of Nicolás Maduro to the present day (2013-2017). Due to the limited time period under consideration and its proximity to the present, the article is guided by a preliminary hypothesis that holds that Venezuelan foreign policy underwent a process of "domestication", that is, an excessive absorption of the internal crisis in behavior external of the State. In reviewing the main facts of the relations between Venezuela and its interlocutors, the actions observed are continuously permeated by domestic conflict. In the first place, we will revew the guidelines in the government program and then, the linkages with the main actors will be addressed. In addition to assessing the validity of the preliminary hypothesis, reviewing the foreign policy proposal will allow to evaluate if the Venezuelan external action has remained close or far from said proposal.

Keywords: Foreign policy; domestication process; crisis; conflict; Venezuela; Nicolás Maduro
\end{abstract}

1 Profesor Asistente en la Facultad Teresa de Ávila, Pontifica Universidad Católica Argentina. Auxiliar docente en Facultad de Ciencia Política y Relaciones Internacionales, Universidad Nacional de Rosario. Correo electrónico: barretomaxi@hotmail.com 


\section{Introducción}

En octubre del año 2012 se llevaron a cabo las elecciones presidenciales para el período 2013-2019, donde Hugo Chávez triunfó frente al candidato de la Mesa de la Unidad Democrática (MUD), Henrique Capriles. Era la cuarta vez que Chávez se sometía a las urnas para acceder al Palacio de Miraflores en el marco de un proceso político que lo tenía como líder indiscutido desde hacía 12 años. La ciudadanía, en esta ocasión, se mostró polarizada. Chávez obtuvo el 55.07 \% de los votos contra el $44.31 \%$ de su contrincante (Consejo Nacional Electoral, 2012). Su gobierno quedó trunco: falleció en marzo de 2013 y accedió a la presidencia interina el vicepresidente, Nicolás Maduro. Conforme a lo estipulado en la Constitución venezolana, el Consejo Nacional Electoral convocó nuevamente a elecciones para el mes de abril. La tendencia electoral se consolidó: Maduro, heredero político de Chávez, ganó por escaso margen (50.61 \% a 49.12 \%) (Consejo Nacional Electoral, 2013). Para algunos actores domésticos como internacionales, estos ajustados guarismos debilitaban en origen la legitimidad del nuevo presidente. Al desconocimiento de los resultados por parte de su oponente, Capriles, se sumaron los reclamos provenientes de España, Estados Unidos (EE. UU.), Francia y Paraguay ${ }^{2}$. El tiempo demostraría que estos reclamos iniciales fueron solo el comienzo y que, cada vez más, la conflictividad interna impregnaría las iniciativas, acciones y respuestas de la política exterior (PE) venezolana. A lo largo de este artículo, a través de una metodología cualitativa, se analizan fuentes primarias ${ }^{3}$ y secundarias ${ }^{4}$ relacionadas con los hitos principales que han nutrido el accionar externo de Venezuela en el período 2013-2017 -en su mayoría citados en el anexo- en pos de identificar a la conflictividad interna como tema recurrente. En el apartado siguiente, se pasa revista de los lineamientos programáticos de la PE previstos para la gestión de Maduro, los cuales, deben ser puestos vis à vis con las secciones sucesivas a efectos de evaluar en qué medida los mismos han podido alcanzarse.

2 Cabe destacar que esta legitimidad "débil" no remitía solamente al pequeño margen de votos que separaba a Maduro del candidato del MUD, sino también a la particular apreciación que diferentes actores tenían del proceso político chavista. En Argentina, Mauricio Macri ganó el balotaje 2015 por tan solo 2.68 \% de los votos; peor aún, en 2016 en Perú, Pedro Pablo Kuczynski ganó por el 0.24 \% de los votos; en Ecuador en 2017, Lenín Moreno ganó por una diferencia del 2.32 \% de los votos y en Brasil se produjo una transición presidencial a través del juicio político a Dilma Rousseff (2016), sin embargo, no se presentaron objeciones similares.

3 Normativa, documentos oficiales de diversas instituciones y declaraciones de actores destacados.

4 Artículos de prensa, trabajos académicos y sitios web. 


\section{Una política exterior preconfigurada}

En el plano de las directrices políticas, Maduro tenía un plan configurado de ante mano. El "Programa de la Patria" (PP), propuesta de gestión difundida por Chávez en la campaña presidencial 2012, fue convertido en ley bajo el nombre de "Segundo Plan Socialista de Desarrollo Económico y Social de la Nación 2013-2019"5(SPSDES), en diciembre de 2013, bajo la presidencia de Maduro. Más allá de que dicha ley aportó muchos detalles y especificaciones al $\mathrm{PP}^{6}$, se estructuró en torno a los 5 grandes objetivos ya presentes en la propuesta de Chávez. Es dable mencionar que, al contrario del reducido espacio que suelen tener las consideraciones de política exterior e internacional en las propuestas de campaña, aquí ocupaban un lugar destacado: 3 de los 5 objetivos articuladores se vinculaban explícita y estrechamente con el plano internacional.

El primero de ellos se denominaba como "La Independencia" y se asentaba sobre el supuesto de haber alcanzado, en la primera década del siglo XXI, la independencia nacional. A partir de entonces, se requería consolidarla, expandirla y evitar el ascenso del denominado "majunchismo", identificado como reproductor de las prácticas de sumisión colonial en base al ideario de Washington; ideario calificado como "imperialista" (PP, 2012, pp. 5-6). Evidentemente, la ley que receptó este objetivo fue redactada sin emplear el vocabulario del discurso chavista, pero conservó las mismas aspiraciones. En sus líneas, se observaba una visión ampliada de la idea de "patria" al asumir que ella es "América" (SPSDES, 2013, p. 4). En este sentido, la conquista de la independencia -como se dijo arriba: asumida como un objetivo ya logrado- se refería a un proceso político más extenso que el circunscripto al interior de las fronteras del Estado, esto es, al denominado "giro a la izquierda", el cual, por entonces mostraba signos de erosión. Implícitamente, se reconocía la importancia de un contexto regional favorable para el proyecto político venezolano. Aunque pa-

5 Publicado en la Gaceta Oficial de la República Bolivariana de Venezuela N..$^{\circ} 6.118$ Extraordinario, 4 de diciembre de 2013. Tuvo como antecedente el denominado "Proyecto Nacional Simón Bolívar" o Primer Plan Socialista del Desarrollo Económico y Social de la Nación para el período 2007-2013.

6 En dicha ley, conservando los lineamientos generales para las políticas públicas, se añadieron programas y acciones concretos para alcanzar a los primeros.

7 Es una palabra acuñada por Chávez con múltiples usos peyorativos para referirse a la oposición política, a quienes asocia con la derecha del país.

8 El "giro a la izquierda" o la "marea rosa" fueron etiquetas con las cuales se buscó caracterizar al proceso político que tuvo lugar a inicios del siglo XXI en América Latina, donde accedieron al poder gobiernos que, si bien con especificidades, se mostraban contrarios al modelo neoliberal promovido por el Consenso de Washington en la década de los noventa. 
rezca una verdad de Perogrullo, un contexto regional afín no es necesariamente una exigencia para la viabilidad de los procesos políticos nacionales: piénsese en el caso de Cuba, por ejemplo. En estrecha relación se presentaba el tercer objetivo. Titulado como "Venezuela: País Potencia", se estructuraba sobre la necesidad de afianzar los lazos con América Latina y el Caribe. En este plano, los esquemas: Alianza Bolivariana para los Pueblos de Nuestra América (ALBA), PETROCARIBE, Comunidad de Estados Latinoamericanos y Caribeños (CELAC) y la Unión de Naciones Suramericanas (UNASUR) aparecían como instancias clave para garantizar una zona de paz regional (PP, 2012, p., 9). Integrando ambos objetivos, la mentada "zona de paz regional" se realizaría a instancias de un escenario regional que sea benevolente al proyecto venezolano. Por último, el cuarto objetivo tenía la aspiración de contribuir a la configuración de un mundo "pluripolar" y "multicéntrico" donde, la "unipolaridad estadounidense", representaba el modelo opuesto (PP, 2012, p., 10).

Aunque este sucinto repaso de los lineamientos programáticos es un ejercicio del plano de las ideas, aporta al entendimiento de la cosmovisión que guiaba a Chávez y de la cual Maduro era su heredero.

Los primeros meses de gobierno de Maduro presentaron un fuerte activismo en el plano internacional en pos de contribuir a afianzar su autoridad, como se dijo, cuestionada por la oposición política y algunos actores internacionales. Horas antes de la juramentación, la UNASUR convocó a una cumbre extraordinaria en Lima para analizar particularmente la situación venezolana-desconocimiento interno al gobierno electo-y dio inicio un breve período de "luna de miel" que se materializó en diversos encuentros de alto nivel. En el mes de junio, Maduro se entrevistó con el Papa Francisco; y tuvo especial interés el encuentro entre el Canciller venezolano Elías Jaua y el Secretario de Estado de EE. UU., John Kerry, en el marco del $43^{\circ}$ inicio de sesiones de la Organización de los Estados Americanos (OEA). Este fue un hecho auspicioso si se piensa que ambos países retiraron sus respetivos embajadores en 2010 y que las relaciones bilaterales estaban tensionadas ${ }^{9}$. En julio, Venezuela asumió la presidencia del Mercado Común del Sur (MERCOSUR) - pese a las objeciones de Paraguay- y tuvo lugar la cumbre con el presidente de Colombia, Juan Manuel Santos.

9 Obama revocó la visa al Embajador venezolano en EE. UU., Bernardo Álvarez, como represalia a la negativa del gobierno de Chávez en aceptar a Larry Palmer como embajador designado (Colmenares, 2018, p. 15) 


\section{"Relaciones de cuarta generación" con Estados Unidos}

En el ámbito de los estudios militares estadounidense ${ }^{10}$ se emplea la idea de "guerra de cuarta generación" para abarcar diversas modalidades que puede asumir la guerra a través de la utilización de estrategias no convencionales de combate. El resultado es la constitución de un campo de batalla sui generis, a menudo, sin ejércitos regulares, y multidimensional: el conflicto se da en los planos político, económico, comunicacional, entre otros. Particularmente, uno de los modos que puede asumir el conflicto, es lo que se conoce como "guerra asimétrica", la cual está dada por la existencia de un actor con recursos de poder muy superiores a los de su oponente y donde, ambos actores - con independencia del "stock de poder"-, se ven obligados a planificar y ejecutar estrategias novedosas ya que la utilización regular del instrumento militar no resulta efectiva. Retomando el sentido "estratégico" que implica para cada uno de los bandos la noción de asimetría citada (y no solo la mera diferencia material; lo cual tendría poca fuerza explicativa ya que las relaciones sin asimetría resultan extrañas), las relaciones bilaterales entre Caracas y Washington pueden ser descriptas en el período bajo estudio como "relaciones asimétricas" o de cuarta generación" El abismal diferencial de poder entre ambos países, entre los años 2013-2017 no se ha traducido en la imposición de la voluntad de EE. UU., la cual se ha visto muchas veces obstaculizada o definitivamente neutralizada.

Ahora bien, aunque el encuentro Jaua-Kerry sonaba esperanzador, el signo que adquirirían las vinculaciones de Venezuela con EE. UU. era, prácticamente, una profecía autocumplida. El PP lo explicitaba de ante mano. El $60 \%$ de los objetivos del plan de gobierno no eran compatibles con el ideario de Washington. Tanto la independencia venezolana y regional como las aspiraciones para con la estructura internacional no podrían concretarse sin objetar a la Casa Blanca. En septiembre de 2013, se inauguraron oficialmente las relaciones conflictivas, tras la aplicación de una medida de reciprocidad por parte del Departamento de Estado de EE. UU., en respuesta a la expulsión de tres funcionarios de su embajada en Venezuela, acusados por Maduro de conspirar contra el gobierno. Sin embargo, la situación crítica se produjo a través de la Orden Ejecutiva 13692

10 Véase El rostro cambiante de la guerra: hacia la cuarta generación, William Lind et al, (1989).

11 Nótese que no se emplea la idea de "guerra" para evitar confusiones con el concepto del campo militar y para describir, con mayor nitidez, relaciones tensionadas entre unidades estatales con -poder dispar- en múltiples planos, incluyendo el militar, aunque se excluya la posibilidad de un uso convencional del instrumento castrense. 
en marzo de 2015, dictada por Barack Obama, donde se declaró emergencia nacional frente a la "unusual and extraordinary threat to the national security and foreign policy of the United States" representada por Caracas. Este decreto se fundamentaba en la ley para la Defensa de Derechos Humanos en Venezuela, sancionada por el legislativo estadounidense en 2014, que habilitaba las sanciones a individuos - especialmente funcionarios- acusados de ser responsables de violaciones a los Derechos Humanos (DD. HH.) en Venezuela. En este contexto, es interesante poner de relieve una situación que se mantendrá a pesar de los progresivos cortocircuitos entre Caracas y Washington: la relación comercial petrolera. Para el año 2016, Venezuela continuaba siendo el tercer origen de las importaciones estadounidenses de crudo y este último el principal destino para Caracas (Faus, 2017).

Aunque en perspectiva, la llegada de Trump (noviembre de 2016) supuso una continuidad -y consolidación- de la conflictividad en las relaciones bilaterales debido a que las posiciones de ambos estados se han radicalizado, cabe destacar que tuvieron una brevísima luna de miel. Dado que Trump, durante la campaña, recurrió a un discurso que lo exponía como el candidato antiglobalización y anti-establishment (proteccionismo, nacionalismo, interpelación a las clases trabajadoras, crítica a los medios de comunicación, entre otros), es probable que los asesores de Maduro hayan visto allí una oportunidad estratégica para intentar modificar el contexto de las desavenencias entre ambos países (Colmenares, 2018, p. 18). En abril de 2017, se conoció de manos de la Comisión de Elecciones Federales (FEC, por su sigla en inglés) que la filial de PDVSA $^{12}$ en EE. UU. (Citgo Petroleum Corporation) aportó medio millón de dólares para la ceremonia de asunción de Trump, tal como figura en el Report of Donations Accepted (2017). Asimismo, una vez asumido Trump como presidente, la PE de Caracas asumió un carácter pragmático: "Aquellas proclamas incendiarias en las que Maduro acusaba al país del norte de ser una potencia imperial y querer aplastar los movimientos progresistas de Latinoamérica y el mundo, cambiaron por un discurso más conciliador y favorable" (Colmenares, 2018, pp. 18-19) ${ }^{13}$.

Sin embargo, una mera observación de lo que sería el gabinete de Trump, aportaba algunos indicios de que las relaciones no serían las mejores. Por ejemplo, en 2015 se conoció un documento titulado Operación Venezuela Freedom-2, que estaría basado en un plan elaborado por el general John Kelly, nombrado por Trump al frente del Departamento de Seguridad Nacional, cuyo antecedente era su exposición en marzo del mismo año ante la Comisión de Servicios Ar-

12 Petróleos de Venezuela S. A.

13 Se recomienda acceder al texto de Colmenares (2018) citado en este artículo, debido a que abunda en mayores detalles sobre el interés venezolano en mejorar las relaciones con EE. UU., en los albores de la administración Trump y el fracaso de esta empresa. 
mados del Senado (Kelly, 2015); y que apuntaba una serie de recomendaciones en rechazo al gobierno de Caracas.

Dos hechos pusieron fin a la luna de miel (Colmenares, 2018, p. 20). Por un lado, “el 13 de febrero de 2017, cuando el gobierno estadounidense decidió imponer sanciones al vicepresidente de Venezuela Tareck El Aissami, al que acusaba de tener vínculos con el tráfico internacional de drogas" (Colmenares, 2018, p. 20). Por otro lado, y en coherencia con el estilo político de Trump, a través de Twitter cuando el presidente exigió la liberación del opositor Leopoldo López: "Venezuela should allow Leopoldo Lopez, a political prisoner \& husband of @liliantintori (just metw/@marcorubio) out of prison immediately" (Trump, 2017a).

Desde allí la tensión fue en ascenso. En el plano discursivo, tanto Trump como el vicepresidente, Michael Pence, se han aproximado al empleo de nociones como rogue state para referirse al gobierno de Maduro. Pence, en un encuentro con la presidenta de Chile, Michele Bachelet, manifestó: "Closer to home, here in our hemisphere, there is another rogue regime. And a tragedy of tyranny is playing out before our eyes in Venezuela" (Pence, 2017). Las sanciones impuestas a funcionarios venezolanos y a sus familiares días después, le dan cuerpo a esta idea ya que se asocian con las medidas que históricamente se han tomado ante situaciones similares (Trump, 2017b). Finalmente, la escalada ha adquirido una impronta tal que, de acuerdo a lo sostenido por Víctor Mijares (2018), "Venezuela ha entrado oficialmente en las líneas de tensión geoestratégicas de las tres mayores potencias actuales". La nueva National Security Strategy (NSS) (NSS, 2017), en su sección para el "Hemisferio Occidental", ha identificado a Venezuela como una amenaza no solo por su crisis interna, sino también por el respaldo que recibe de China y Rusia, actores reconocidos en dicho documento como retadores hegemónicos de EE. UU. (NSS, 2017, p. 51) ${ }^{14}$.

A pesar de todo lo dicho, el aumento de la conflictividad bilateral no se ha traducido en la inclinación de la balanza hacia uno u otro actor. Retomando las ideas planteadas al inicio de este apartado, con el ascenso de Trump, el carácter "asimétrico" de las relaciones se ha acentuado y, aunque parezca ficcional, Venezuela ha ejercido un "veto tácito" al empleo del poder bélico por parte de la Casa Blanca. Ello ha conducido a ambos actores a ejecutar estrategias atípicas,

14 "El origen de esta riesgosa situación se remonta a la estrategia de política exterior y de seguridad de Hugo Chávez. El padre de la Revolución Bolivariana se planteó tempranamente como meta contribuir con un "mundo pluripolar" (sic). Venezuela se comportó asertiva y prolongadamente como un jugador geoestratégico, es decir, como un actor internacional capaz de modificar las condiciones del sistema internacional. Sin embargo, su verdadera naturaleza es la de un pivote geopolítico, o una unidad internacional cuya importancia no se deriva de su poder, sino de su localización y recursos naturales (ambos apreciados y explotables por los verdaderos jugadores geoestratégicos)" (Mijares, 2018). 
donde parecería que el Palacio de Miraflores ha usufructuado mejor la asimetría. En tal sentido, Trump no logró apoyos para una eventual "intervención militar" 15 y vio neutralizadas sus acciones en la OEA, tal como veremos en el apartado siguiente. Si bien las sanciones económicas, como la prohibición para los ciudadanos estadounidenses de emitir o comprar deuda venezolana, tienen su efecto al limitar, por ejemplo, la captación de divisas por parte de Maduro; podrían encontrar un límite generado por la asimetría de poder. En esta línea opina David Smilde en The New York Times:

(...) Estados Unidos y sus aliados deben evitar la tentación de ampliar el espectro de las sanciones. Ensanchar las sanciones económicas para incluir, por ejemplo, un embargo petrolero haría más daño a la población, que apenas se sostiene. Y esa ampliación de sanciones enfocadas, que buscan causar una división entre los sancionados y los no sancionados, anularía el efecto: si casi todos están sancionados, más bien consolidará la unidad del gobierno de Maduro. (Smilde, 2018)

Asimismo, cabe mencionar que otro elemento que ha limitado a favor de Caracas las sanciones vociferadas por Trump, es la ya mencionada interdependencia petrolera, no solo en términos comerciales. Así, a mediados de 2017, la petrolera venezolana Citgo logró quedar exenta del conjunto de sanciones impuestas por Trump -con motivo de la convocatoria a una Asamblea Constituyente por parte de Maduro- debido a la participación de la Citgo en la economía de EE. UU. a través de tres refinerías y casi seis mil estaciones de servicio. Por otro lado, la interdependencia hidrocarburífera no solo se divisó en esta firma: también las refinerías de petróleo a lo largo de la costa del golfo estadounidense que procesan crudo venezolano y representan casi el $10 \%$ de las importaciones estadounidenses, alertaron sobre los efectos negativos de aplicar determinadas sanciones: "Bloquear las importaciones podría causar un gran daño a esta industria y cargar a los contribuyentes estadounidenses con el costo" (Gardner, 2017). En el mes de agosto de 2017, pese a que las sanciones en cierta medida comenzaron a afectar a la Citgo al impedir la transferencia de sus dividendos hacia Caracas, el comercio no fue prohibido (Lawder y Ulmer, 2017). En este marco, tal como lo expresa Dallen (2017) "la gente puede sorprenderse al saber que EE.UU. ha estado financiando a la administración de Maduro".

\section{Relaciones con actores e instituciones regionales y continentales}

15 Países y organismos como Chile, Colombia, Perú, OEA, MERCOSUR e, inclusive el MUD, se distanciaron de los dichos de Trump sobre una posible intervención militar (Oliphant y Buitrago, 2017). 
Como se sabe, la apreciación de Chávez sobre la OEA no había sido la mejor. Su opinión estaba impregnada por consideraciones clásicas del período de guerra fría, muy similares a la postura históricamente sostenida por $\mathrm{Cuba}^{16}$. El gobierno de Miraflores del período bajo estudio, mantuvo esta lectura y decidió, en marzo de 2017, iniciar el proceso de "autoexclusión” del organismo. Aunque es cierto que, como veremos en seguida, la OEA ha sido el espacio continental que más altercados políticos-discursivos ha tenido contra Venezuela, la visión en clave de "guerra fría" no resulta adecuada en sentido estricto ya que EE. UU. ha encontrado obstáculos para extender, a la gran mayoría de los estados, su perspectiva. Por otro lado, aquellos esquemas más amigables para el proceso venezolano (ALBA, UNASUR), aunque han servido para amortiguar las fricciones regionales, no se han presentado como instancias efectivas de respaldo. El propio MERCOSUR que, para el inicio de la administración Maduro, era caracterizable como un esquema no hostil para Caracas, con el paso de los años se fue convirtiendo en un lugar discrepante. Al inicio del gobierno, Argentina, Brasil y Uruguay con Cristina Fernández, Dilma Rousseff y José Mujica, respectivamente, condujeron el bloque como aliados del Palacio de Miraflores. Las transiciones presidenciales, fundamentalmente, en Argentina (2015) y Brasil (2016) comenzaron a torcer dicha posición. En el caso de Argentina es ilustrativa la posición de ambigüedad que, desde el inicio de la gestión Macri, asumió el posicionamiento de Buenos Aires respecto a Caracas (hasta mayo de 2017). Dada la carrera política hacia la Secretaría General de las Naciones Unidas de la entonces Canciller argentina Susana Malcorra, la crítica hacia Maduro fue en gran parte discursiva. Venezuela ocupaba el asiento no permanente en el Consejo de Seguridad, por lo cual, era un voto que Malcorra necesitaba. En el seno de la OEA la posición argentina se atenuó y no apoyó la activación de la Carta Democrática Interamericana (CDI), tal como lo demandaba la oposición política venezolana a Maduro (Allup, 2016).

La ola de protestas de enero y febrero de 2014 - con un terrible saldo de muertos y heridos- puede considerarse como el primer test case del accionar del sistema de instituciones en el nivel regional y continental frente a Venezuela. En términos generales, salvo el caso del MERCOSUR, el comportamiento observado será similar a lo largo de los años. Retomando lo dicho, aunque la OEA se presentaba para Caracas como una instancia poco amistosa (Chávez había sido un fervoroso promotor de la CELAC, como espacio sin la presencia de EE. UU. y Canadá), en esta primera prueba, la diplomacia de Maduro fue efectiva. El blo-

16 Por ejemplo, ya en agosto de 1960, en el discurso pronunciado en el acto de clausura del Primer Congreso Latinoamericano de Juventudes, Fidel Castro comentaba: “¿Y qué quieren los yankis? ¿Quieren decir que nosotros desacatamos la OEA? Pues si quieren decirlo, magnífico, que digan lo que nosotros decimos: que tienen la OEA como un instrumento para impedir revoluciones en América" (Castro, 1960). 
que quedó neutralizado. La declaración 51 (1957/14) del Consejo Permanente y las reservas de Panamá y EE. UU. ante la mera actitud declarativa del organismo lo ejemplifican. El sistema de alianzas venezolanas constituido por los países miembros de ALBA y La Comunidad del Caribe (CARICOM) fue decisivo. CELAC, UNASUR y MERCOSUR, se comportaron prácticamente de la misma manera con laxos llamados al diálogo, aunque, UNASUR dispuso la creación de una comisión de cancilleres para apoyar y asesorar el diálogo político.

La alianza con los países del ALBA y CARICOM se había visto fortalecida en el período de gobierno de Chávez sobre la base de la diplomacia petrolera, propia de la coyuntura de los elevados precios internacionales del petróleo. El PP, jerarquizaba a estos bloques, sin embargo, en un contexto de depreciación de los precios de los hidrocarburos, perdurar el lazo era un desafío para Maduro ${ }^{17}$. La tendencia a la baja de aquellos precios, ilustrada por el gráfico a continuación, se tradujo en un significativo descenso de las divisas con las cuales el gobierno venía sosteniendo su activa PE.

\section{Evolución del precio del petróleo, OPEP 2018}

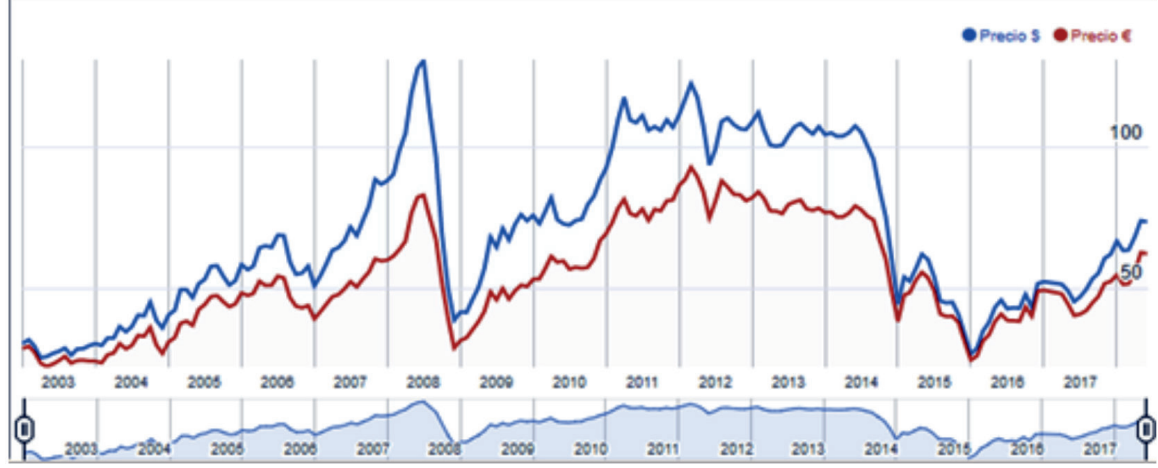

Figura 1. Evolución precios internacionales del petróleo (2003-2013).

Fuente: "Precio del petróleo 2018"

Si se observa el gráfico hacia el presente, nuevamente el precio del petróleo muestra una tendencia al alza; lo que es una buena noticia para el gobierno en el sentido mencionado arriba: acceso a divisas. Miranda Delgado (2016, p. 334) plantea una tesis en este sentido: "el nivel de activismo de la política exterior de Venezuela depende del mercado petrolero y, especialmente, de los precios internacionales dictados por este mercado, es decir, a mayores precios del petróleo mayor activismo de la política exterior de Venezuela, y viceversa".

17 En este contexto, Venezuela jugó su carta proponiendo reducir la producción petrolera para defender los precios. Véase: $B B C$, febrero 2016. Disponible en: http://www.bbc.com/mundo/noticias/2016/02/160216_economia_paises_productores_petroleo_pueden_subir_precios_lf 
Efectivamente, ante la caída de los precios internacionales del petróleo los acuerdos de cooperación petrolera con países del Caribe y Centroamérica se vieron afectados. El caso de Cuba es representativo: a la paralización de los proyectos de construcción de instalaciones petroquímicas, se sumaron restricciones al propio suministro de petróleo - que a su vez era subsidiado- (Martín, 2016). De recibir 100000 barriles de crudo a finales del 2013 bajo esa iniciativa, en 2015 pasó a recibir apenas unos 35000 (Romero, 2017). No obstante, para algunos analistas, es esperable que Miraflores se esfuerce en mantener PETROCARIBE pese a las pérdidas, ya que el bloque se creó por razones políticas, como herramienta efectiva de influencia para las naciones caribeñas, principalmente (Romero, 2017). En términos políticos, a pesar de que el hecho se ha extendido a otros países integrantes de PETROCARIBE, el respaldo político hacia Caracas no se ha perdido ${ }^{18}$. Ello es lo que ha ocurrido en el seno de la OEA. Los países caribeños han apoyado el principio de no intervención esgrimido por Maduro.

En relación con PETROCARIBE, hay que destacar que México ha empezado a diseñar un plan para reemplazar el programa venezolano de petróleo, mediante una propuesta propia de crudo y productos petroleros de bajo costo a los clientes de aquel esquema en el Caribe y Centroamérica. Un plan que, según se dice, parece contar con el beneplácito y el estímulo del gobierno de EE. UU. (Stargardter, 2017)

Hacia mediados de 2016, la situación al interior de la OEA empeoró como reflejo de la crisis doméstica venezolana en ascenso. A inicios de ese año, el Tribunal Supremo de Justicia (TSJ) declaró en desacato a la Asamblea Nacional (AN) - de extracción mayoritariamente opositora- por hacer jurar a tres diputados del Estado de Amazonas que, en su entendimiento, no estaban habilitados. En mayo, el Secretario General de la OEA, Luis Almagro, presentó ante el Consejo Permanente un extenso y duro informe contra Venezuela (Almagro, 2016), que evidenciaba su postura de manera muy clara. En la acostumbrada cita de la Asamblea General en el mes de junio, se vaticinaba que el fantasma (activación) de la CDI rondaría, motivado más por el empeño de Almagro que por el grueso de los Estados miembros. Por su parte, la posición de Venezuela era encauzar un diálogo en los términos propuestos por UNASUR, con el liderazgo de los expresidentes: José Luis Rodríguez Zapatero (España), Martín Torrijos (Panamá) y Leonel Fernández (República Dominicana).

18 Ver más adelante que, independientemente del apoyo político otorgado al gobierno de Maduro en la crisis institucional, los países caribeños no se han comportado del mismo modo en la disputa con Guyana. 
El período 2017 de la Asamblea General fue casi idéntico. Almagro fracasó en marzo en su empresa de activar la CDI, a la par que la conflictividad al interior de Venezuela no dejó de aumentar. El TSJ asumió las competencias de la propia AN (marzo), la cual, días atrás había solicitado la aplicación de la mencionada CDI y la suspensión del país en la OEA. En abril, el TSJ devolvió el poder a la AN, sin embargo, en junio el equilibrio de cristal se rompió. El gobierno de Maduro convocó a una Asamblea Constituyente, que fundaría un nuevo orden. La decisión debilitó los apoyos internacionales con que contaba el gobierno. Por ejemplo, puso fin a la situación de no intervención del MERCOSUR. Uruguay que venía siendo el contrapeso a favor de Maduro, no fue capaz de soportar las presiones de Argentina, Brasil y Paraguay. Venezuela fue suspendida del bloque por unanimidad a causa de una "ruptura del orden democrático" tal como reza la decisión del 5 de agosto de $2017^{19}$ y de conformidad con el segundo párrafo del artículo 5. ${ }^{\circ}$ del Protocolo de Ushuaia, sobre Compromiso Democrático en el MERCOSUR, suscrito el 24 de julio de 1998. Cabe destacar que, desde entonces, Uruguay ha mantenido cierta distancia relativa del gobierno de Maduro, aunque en ocasiones muestra posiciones -al menos simbólicas- de solidaridad con Caracas. Montevideo se opuso a la conformación del "Grupo de Lima" - mencionado luego- y mostró su rechazo al intento de atentado contra Maduro de agosto de 2018. De todas maneras, la convocatoria a una Asamblea Constituyente terminó por aislar a Maduro en la subregión latinoamericana ya que solo conservó el apoyo de Bolivia -el respaldo de Ecuador se había perdido con la llegada de la administración de Lenin Moreno (Oliva, 2017, p. 2). Ante la parálisis de la OEA, se conformó en agosto el "Grupo de Lima", instancia ad hoc nacida de la "Declaración de Lima" y con un posicionamiento crítico hacia el gobierno venezolano. Lima -casi haciendo honor al nombre del grupo- ha elevado su oposición discursiva hacia Caracas: es su rechazo de recibir a la delegación venezolana en territorio peruano, sede de la VIII Cumbre de las Américas, un ejemplo de esta conducta. Claramente, en ocasión de las elecciones presidenciales que consagraron nuevamente como presidente a Maduro, en mayo de 2018, el "Grupo de Lima” desconoció los resultados.

\section{Relaciones con actores europeos}

En el otro lado del océano Atlántico, la convocatoria a la Constituyente fue el motivo propicio para que las posiciones más duras al interior de la Unión Europea (UE) encontraran asidero. Hasta la fecha, aunque el bloque había ejercido presión política por la liberación de los presos políticos, el respeto al calendario

19 Venezuela ya había sido suspendida en el ejercicio de sus derechos como Estado parte del MERCOSUR anteriormente, en diciembre de 2016, en virtud del incumplimiento de compromisos asumidos en su Protocolo de Adhesión. 
electoral y a la Asamblea Nacional y la apertura de un canal humanitario; la representante de la UE para la Política Exterior, Federica Mogherini, también había respaldado el diálogo iniciado entre el gobierno de Maduro y la oposición a fines de 2016. A instancias del Vaticano, en octubre de 2016, se emprendió una mesa de diálogo que encontraba sus antecedentes en las iniciativas de UNASUR de 2014, 2015 y mayo de 2016 (delegación de cancilleres y diálogo encabezado por tres expresidentes, respectivamente), pero que terminó fracasando pocos meses después.

Para junio de 2017, el clima de animosidad que se vivía en el plano doméstico venezolano, comenzó a reflejarse en la UE. Se rechazó de lleno la convocatoria a una Constituyente, y se emprendió el camino de las sanciones: embargo de armas y materiales utilizables en actividades de represión interna; inmovilización de activos y la prohibición a determinados funcionarios para viajar (Consejo Europeo, 2017). Este fue el contexto propicio en el que la posición del gobierno español contra Caracas tomó impulso. Cabe destacar que aquel país, desde el primer día de gestión de Maduro había entrado en contradicción con Venezuela. Para enero de 2018, ambos Estados habían expulsado sus respectivos embajadores. Hacia mayo del mismo año, la situación empeoró a raíz de las elecciones presidenciales ya mencionadas en Venezuela, donde el -ahora destituido- presidente del gobierno español, Mariano Rajoy, encabezó el rechazo en el nivel europeo hacia las elecciones.

\section{Relaciones con China y Rusia}

China y Rusia aparecían implícitamente mencionados en el PP, en la aspiración de contribuir a la configuración de un mundo "pluripolar" y "multicéntrico". Ambos países habían tenido un papel protagónico en la primera década del 2000 , liderando el the rise of the rest. Sin embargo, la administración de Nicolás Maduro inició en otro contexto. El proceso de ascenso de los poderes emergentes estaba menguado y, peor aún, Venezuela estaba inmersa en una crisis fenomenal. En este sentido, China y Rusia antes que aliados en la construcción de un nuevo orden, pasaron a constituir un sostén fundamental para Caracas.

En términos políticos-discursivos, Rusia se ha manifestado menos cautelosa que China al momento de dar a conocer su postura sobre la situación venezolana. Frente a la objeción internacional que se generó en torno a la convocatoria de una Convención Constituyente (2017), Moscú se mostró favorable e instó a la comunidad internacional a abandonar aquella posición, caracterizada como "destructiva". Sin embargo, es interesante destacar que la cautela de China, ha devenido en un apoyo material más generoso que el ruso; lo 
que, en consecuencia, le ha otorgado a Pekín más influencia sobre el Palacio de Miraflores. China tiene dinero para prestar, y controla instrumentos financieros, proyectos de obras y flujos de bienes importantes para la supervivencia del gobierno (Ellis, 2017, p. 2). En términos materiales, aunque el apoyo ruso ha sido menor, no deja de ser relevante. En este caso, el respaldo se ha concentrado principalmente en el rubro de la venta de armamento, ejercicios militares e inversiones en el sector petrolero (2017, p. 8). La vinculación en estas áreas, usualmente identificadas como sectores estratégicos, ha contribuido a potenciar el carácter "bipolar" del comportamiento ruso en Venezuela. Recreando el clima de "guerra fría", la proyección militar rusa en Venezuela suele ser vista como una provocación para EE. UU. Precisamente, esta ha sido la clave en la que Washington se ha planteado la presencia de Moscú en el área:

Russia continues its failed politics of the Cold War by bolstering its radical Cuban allies as Cuba continues to repress its citizens. Both China and Russia support the dictatorship in Venezuela and are seeking to expand military linkages and arms sales across the region. The hemisphere's democratic states have a shared interest in confronting threats to their sovereignty. (SSN, 2017, p. 51)

En relación con la citada Citgo, es interesante ilustrar la situación de triangulación y temor "bipolar" que se produjo en este período. Recientemente, la petrolera rusa Rosneft otorgó un préstamo a la Citgo que podría entregarle a Rusia el control del $19 \%$ de las refinerías en EE. UU., dado que se empleó como garantía del empréstito al $49.9 \%$ de las acciones de la empresa. La crisis económica en Venezuela podría llevar a la Citgo a incumplir su deuda, y ello colocaría a Rosneft en condiciones de controlar tres de las refinerías más grandes y sofisticadas de EE. UU., además de tres grandes oleoductos, y docenas de terminales de combustibles (Brito, 2017). Sin dudas, la aproximación de Rusia a Venezuela en estos temas es un efecto colateral de las sanciones petroleras estadounidenses que obligan a Venezuela a acercarse a otros actores. Particularmente, Rusia ha aprovechado la crisis generalizada para comprar activos petroleros venezolanos, de alto valor (estratégico) a largo plazo.

\section{Relaciones con el exterior cercano}

Finalmente, mención especial merecen los países emplazados en lo que la geopolítica venezolana denomina como los "frentes geopolíticos venezolanos". Tal como puede verse en el mapa, incumbe a países que comparten una frontera común, y a los cuales la proximidad territorial los hace "geográficamente 
solidarios” entre sí. En este caso, corresponde a países que difícilmente pueden permanecer indiferentes ante la crisis venezolana.

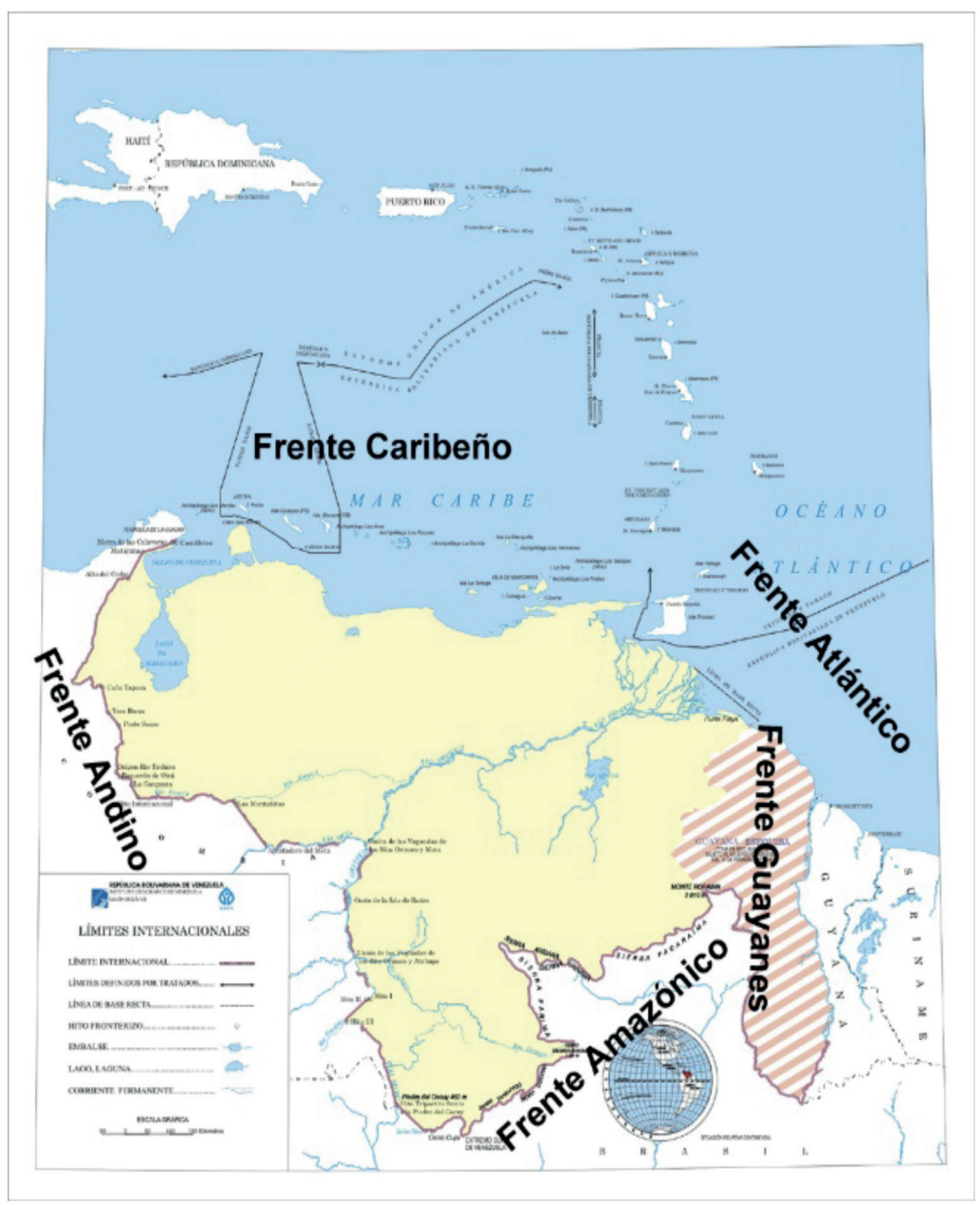

Figura 2. Frentes geopolíticos venezolanos.

Fuente: Instituto Geográfico de Venezuela "Simón Bolívar”, 2018.

En relación con el frente andino, cabe destacar el aspecto conflictivo que han adquirido - nuevamente ${ }^{20}$ - las relaciones bilaterales desde la llegada de Maduro. Colombia es el principal país afectado por el proceso migratorio generado

20 Bajo la presidencia del colombiano, Juan Manuel Santos, se restablecieron las relaciones diplomáticas, obstruidas en el año 2010, mientras gobernaba Álvaro Uribe. 
por la crisis venezolana. Se calcula que, para fines de 2017, residían con visas de extranjería debidamente expedidas, alrededor de 50000 venezolanos, y otros 70000 con visas válidas de tres meses. Asimismo, se estipula que más de 160 000 que en algún momento tuvieron esa visa temporal, se encuentran viviendo en territorio colombiano ilegalmente. Por otro lado, de manera informal, serían al menos 140000 los que han cruzado la larga frontera común (Leizaola, 2017, p. 2). Dada la complejidad de esta situación, en numerosas ocasiones las fronteras se han cerrado unilateralmente y se han generado momentos de máxima animosidad como, por ejemplo, en 2016 cuando Venezuela expulsó a 1000 colombianos (Bocanegra, 2015). Hechos similares se han registrado en el frente amazónico y caribeño (Brasil, Aruba, Curazao y Bonaire).

Por otro lado, desde el punto de vista estratégico, las relaciones se han tensionado por varios motivos. La tradicional cercanía de Bogotá con la Casa Blanca es vista con preocupación desde Caracas. Para el año 2018, se prevé que Colombia recibirá una ayuda de 251 millones de dólares. El $75 \%$ correspondiente al programa de Seguridad y Paz, y poco más de 44 millones para programas de Democracia, Derechos Humanos y Gobernabilidad. En este plano, aunque Perú no forma parte de los aludidos frentes, el último tiempo ha representado para Venezuela una preocupación similar dado que se encuentra entre los principales receptores de asistencia militar por parte de EE. UU. La asistencia para 2018 será de 49 millones de dólares, siendo 48 millones los destinados a Seguridad y Paz. Este proyecto comprende la lucha contra el narcotráfico en una zona que no ha sido enteramente controlada por el Estado peruano y con remanentes de la guerrilla de Sendero Luminoso que, junto con las Fuerzas Armadas Revolucionarias de Colombia, son consideradas por el Comando Sur de EE. UU. como fuente potencial de terrorismo. Todo ello, en un contexto en el que se ha incrementado la instalación de bases militares en la selva peruana y donde la animosidad entre Maduro con el nuevo presidente del Perú -ahora fuera del cargo por su renuncia-, ha sido una nota distintiva (García Fernández, 2018).

En el mismo ámbito, la aspiración colombiana a profundizar la cooperación con la Organización del Tratado del Atlántico Norte (OTAN) ha generado roces similares. En 2013, se firmó un fallado acuerdo con aquella organización que luego fue declarado inconstitucional por la Corte Constitucional colombiana en 2015. Los rumores traen consigo la posibilidad de renegociar dichos acuerdos: la incorporación colombiana a la OTAN, en mayo de 2018, como "socio global" va en esa dirección.

Por último, en el frente guayanés, la relación con Guyana suma un condimento tradicional a las relaciones bilaterales: ambos países comparten una disputa territorial por el Territorio del Esequibo o Región del Esequibo, espacio ac- 
tualmente administrado por Guyana y reclamado por Venezuela. Aunque la problemática tiene larga data, una serie de hechos han alimentado la tensión bilateral a partir de 2013, adquiriendo rasgos que no se habían observado en todo el período chavista.

El 2013 fue un periodo en el que Caracas y Georgetown parecieron comprometerse con la búsqueda de una solución a la disputa. El espacio marítimo proyectado por el territorio del Esequibo, era un buen lugar para emprender el camino, dado que las actividades pesqueras causaban el grueso de los altercados. En efecto, se acordaron explorar mecanismos para la delimitación marítima y debido al breve plazo que se establecía para el inicio de los trabajos técnicos, todo indicaba que en un período razonable se podría saldar las diferencias. Sin embargo, el acuerdo quedó en el papel.

Por su parte, Venezuela asumió una conducta dilatoria mientras que Guyana prosiguió con una política de hechos consumados: reactivó su solicitud en la Organización de las Naciones Unidas (ONU) para ampliar la plataforma continental de 200 a 350 millas, firmó acuerdos con transnacionales petroleras de concesión y exploración hidrocarburífera, incluso, declaró la posibilidad de construir una represa junto a Brasil en esta misma zona. En mayo de 2015, la problemática se agudizó por el descubrimiento de petróleo. Solo entonces, Caracas tomó una actitud más activa. Maduro aprobó el decreto 1787 que creó las Zonas Operativas de Defensa Integral Marítima e Insular (ZODIMAIN) y que, para peor, estaba mal redactado ya que incluía coordenadas correspondientes no solo a la proyección marítima del territorio Esequibo, sino a la de Georgetown y parte de Surinam, donde Venezuela nunca ha tenido aspiraciones territoriales. Si bien una semana después el decreto fue sustituido por el 1859, el vínculo prosiguió tensionado. A la vez, la Canciller venezolana había emprendido una gira por el Caribe en pos de difundir la posición del gobierno, cuya estrategia diplomática fue criticada por centrarse en acusar a Guyana de tener una "agenda oculta" con las empresas trasnacionales y de crear una división y desestabilización regional (Romero, 2015). A diferencia de lo ocurrido en el seno de la OEA, en esta cuestión, la comunidad caribeña apoyó a Guyana, llegando a solicitar a Maduro la revisión del decreto que creaba las ZODIMAIN.

Finalmente, conforme a lo estipulado en el Acuerdo de Ginebra de $1966^{21}$, el Secretario General de la ONU, Ban Ki-moom, antes de finalizar su mandato, emitió un comunicado que informaba que el proceso de buenos oficios -elegido desde fines de los 80 como método para intentar resolver la disputa- continuaría por un último año hasta el final de 2017. Asimismo, advirtió que, de no

21 Tratado en el cual se enmarcan desde 1966 los intentos de resolución del conflicto. 
existir un avance significativo para llegar a un acuerdo total, se recurriría a la Corte Internacional de Justicia (CIJ) como método, a menos que los gobiernos conjuntamente se opusieran. Efectivamente, a fines del año 2017, el nuevo Secretario General, Antonio Guterres, informó su decisión de someter la disputa a la CIJ, al vencer el plazo y no haber avances significativos. La comunicación fue condenada por Caracas.

\section{Conclusiones}

Advirtiendo que este trabajo es un acercamiento preliminar y que no pretende ser exhaustivo, los hitos narrados son un insumo para sostener la hipótesis de la domesticación de la PE venezolana. Si bien, toda PE está permeada por factores provenientes del plano interno -incluso hay autores que definen a dicha política como una herramienta puntual para resolver problemáticas de ese orden-, la especificidad del caso remite a que la crisis interna es la fuente que aporta el grueso de los incentivos que nutren las acciones exteriores del país. Asimismo, esta situación se replica en el plano reactivo, pues Caracas debe emitir respuestas a demandas del exterior motivadas, en su mayoría, por la conflictividad doméstica. Así, las iniciativas ALBA y PETROCARIBE, que otrora fueron ejemplos del activismo internacional de Venezuela y del liderazgo en la subregión andina y caribeña; ahora se dirigen a apuntalar al gobierno en su disputa política con los sectores de la oposición. Del mismo modo, las expulsiones del personal de las embajadas o la salida del país de la OEA, son respuestas a demandas de actores internacionales impregnadas por el conflicto interno. Por añadidura, la domesticación de la PE se ha vuelto de tal envergadura que, asuntos de larga data como la controversia por el territorio Esequibo - en nada vinculados con la crisis doméstica actual-, también se han visto salpicados por ese factor. En este sentido, se manifiestan algunos críticos al gobierno al argumentar que, el desenlace actual del conflicto se debe a la poca atención que las autoridades han podido dedicar al mismo, dada la situación política, económica y social.

Por último, aunque los sucesos citados muestran el asedio que sufre el gobierno de Nicolás Maduro, en Venezuela parece reproducirse la idea de "un empate hegemónico", que José Natanson (2017, p. 2) emplea para el caso latinoamericano. En los términos clásicos de Juan Carlos Portantiero, Natanson describe a la realidad regional como una situación en donde dos fuerzas en disputa tienen suficiente energía como para vetar los proyectos elaborados por la otra, pero donde ninguna logra reunir los recursos necesarios para asumir el liderazgo por sí sola. Si pensamos que, en el contexto de una crisis fenomenal ${ }^{22}$, el go-

22 Menciónese, por ejemplo, una caída acumulada del PIB del 32.5 \% para el período 2013-2017; niveles de inflación altísimos (cerca de $1200 \%$ ); reservas internacionales que descienden a los rangos más 
bierno ganó a fines de 2017 el $91 \%$ de los municipios y obtuvo casi el $70 \%$ de los votos; y que, en el ámbito externo, ni siquiera EE. UU., ha podido imponer su voluntad -la cual, de hecho, se ha visto muchas veces obstaculizada o definitivamente neutralizada-, quizás esta conjetura no sea del todo descabellada.

\section{Referencias}

Acuerdo para resolver la controversia sobre la frontera entre Venezuela y Guayana Británica, Secretaría de las Naciones Unidas [Acuerdo de Ginebra], (1966). Disponible en: https://es.wikisource.org/wiki/Acuerdo_de_Ginebra_ de_1966 (Consultado el 20 de junio de 2018).

Allup, H. (2016). @hramosallup [Tweet de la cuenta verificada de Allup], (2016). Disponible en: https://twitter.com/hramosallup/status/738144441618161664 (Consultado el 14 de junio de 2018)

Almagro, L. Organización de los Estados Americanos. (2016). Disponible en: http://www.oas.org/documents/spa/press/OSG-243.es.pdf (Consultado el 14 de abril de 2018).

Bloqueo de los activos y suspensión de la entrada a ciertas personas que contribuyen a la situación en Venezuela, Casa Blanca, [Orden Ejecutiva 13692]. (2015). Disponible en: http://cdn.eluniversal.com/2015/03/09/ordenejecutiva. pdf (Consultado el 20 de junio de 2018).

Bocanegra, N. (2015). "EEUU pide a Colombia y Venezuela resolver diplomáticamente crisis por cierre de frontera", en Reuters. Disponible en: https://lta.reuters.com/article/domesticNews/idLTAKCN0QZ0QV20150830 (Consultado el 14 de junio de 2018).

Brito, V. (2017) "Citgo contrata a firma de exjefe de campaña de Trump para evitar sanciones”, en Diario Las Américas. Disponible en https://www.diariolasamericas.com/eeuu/citgo-contrata-firma-exjefe-campana-trump-evitar-sanciones-n4121316

Castro, F. (1960). "Discurso pronunciado por el Comandante Fidel Castro Ruz, en el acto de clausura del 1. ' Congreso Latinoamericano de Juventudes", en Portal Cuba. disponible en: http://www.cuba.cu/gobierno/discursos/1960/ esp/f060860e.html (Consultado el 30 de agosto de 2018).

bajos en los últimos 20 años (menos de 9800 millones de dólares) y escasez de toda clase de bienes y servicios esenciales (alimentos y medicinas) (Sutherland, 2017). 
Colmenares, A. (2018). "Las relaciones de Venezuela con Estados Unidos en la era de Trump", en Foreign Affairs Latinoamérica, 18:1, pp. 17-24. Disponible en: http://www.fal.itam.mx (Consultado el 19 de junio de 2018).

Consejo Europeo. (2017). Disponible en: http://www.consilium.europa.eu/es/press/ press-releases/2017/11/13/venezuela-eu-adopts-conclusions-and-targetedsanctions (Consultado el 20 de junio de 2018).

Consejo Nacional Electoral. (2012). Disponible en: http://www.cne.gob.ve/resultado_presidencial_2012/r/1/reg_000000.html (Consultado el 9 de mayo de 2018).

Consejo Nacional Electoral. (2013). Disponible en: http://www.cne.gob.ve/resultado_presidencial_2013/r/1/reg_000000.html (Consultado el 9 de mayo de 2018).

Dallen, R. (2017). "Una filial de PDVSA complica los planes de Trump en Venezuela", en El Cronista. Disponible en: https://www.cronista.com/financialtimes/Una-filial-de-PDVSA-complica-los-planes-de-Trump-con-Venezuela-20170726-0049.html (Consultado el 3 de septiembre de 2018).

Ellis, R. (2017). "The Influence of Extra-Hemispheric Actors on the Crisis in Venezuela" [Testimonio], Foreign Affairs Committee, U. S. House of Representatives, Washington.

Faus, J. (2017). "El millonario negocio petrolero de Venezuela con Estados Unidos escapa de las sanciones", en El País. Disponible en: https://elpais.com/internacional/2017/05/29/estados_unidos/1496017333_399364.html (Consultado el 3 de septiembre de 2018).

García Fernández, A. (2018). "FAA en América Latina: armas, capacitación y el cerco a Venezuela", en CELAG.ORG. Disponible en: http://www.celag.org/ ffaa-america-latina-armas-capacitacion-cerco-venezuela/ (Consultado el 10 de marzo de 2018).

Gardner, T. (2017). "Senadores EEUU piden a Trump que evite bloqueo a envíos de petróleo de Venezuela", en Reuters. Disponible en: https://lta.reuters.com/ article/worldNews/idLTAKBN1AR031-OUSLW (Consultado el 3 de septiembre de 2018).

Instituto Geográfico de Venezuela "Simón Bolívar". (2018). Disponible en: http:// www.igvsb.gob.ve/

Kelly, J. (2015). Posture Statement of General John F. Kelly, United States marine corps commander, United States Southern Command [Declaración], Comisión Servicios Armados, Senado EE. UU. Disponible en: http://www.defenseinnovationmarketplace.mil/resources/SOUTHCOM_POSTURE_STATEMENT_FINAL_2015.pdf (Consultado el 10 de junio de 2018). 
Lawder D. y Ulmer, A. (2017). "Sanciones de Trump buscan frenar financiamiento a 'dictadura' en Venezuela", en Reuters, 25 de agosto de 2017. Disponible en: https://lta.reuters.com/article/topNews/idLTAKCN1B5222-OUSLT (Consultado el 3 de septiembre de 2018).

Leizaola Las Heras, T. (2017). "De Venezuela a Colombia. La emigración no se detiene", en Nueva Sociedad, agosto 2017. Disponible en: http://nuso.org/articulo/de-venezuela-colombia/ (Consultado el 19 de junio de 2018).

Lind, W. et al (1989). "El rostro cambiante de la guerra: hacia la cuarta generación", en Marine Corps Gazzete, pp.22-26. (Consultado el 11 de octubre de 2018).

Martín, S. (2016). “Argelia 'rescatará' a Cuba ante la caída en el suministro de petróleo desde Venezuela", en Panam Post. Disponible en: https://es.panampost. com/sabrina-martin/2016/09/08/argelia-rescatara-a-cuba-ante-la-caida-enel-suministro-de-petroleo-desde-venezuela/ (Consultado el 10 de mayo de 2018).

Mijares, V. (2018). "Un riesgo innecesario: Venezuela en el juego de las grandes potencias", en Politika Revista del Centro de Estudios Políticos. Disponible en: https://politikaucab.net/2018/01/09/un-riesgo-innecesario-venezuela-enel-juego-de-las-grandes-potencias/?platform=hootsuite (Consultado el $18 \mathrm{de}$ mayo de 2018)

Ministerio de Asuntos Exteriores de la Federación de Rusia. (2017). Disponible en http://www.mid.ru/ru/foreign_policy/news/-/asset_publisher/cKNonkJE02Bw/content/id/2824984 (Consultado el 20 de abril de 2018).

Miranda Delgado, R. (2016). "Política exterior de Venezuela: el petróleo como variable estructuradora", en Analecta polit, 6: 11. Medellín, Colombia.

Natanson, J. (2017). “Empate hegemónico en América Latina”, en Le Monde diplomatique. Buenos Aires, junio-julio.

National Security Strategy of the United States of America, Casa Blanca [Documento]. (2017). Disponible en: https://www.whitehouse.gov/wp-content/ uploads/2017/12/NSS-Final-12-18-2017-0905.pdf (Consultado el 10 de junio de 2018).

Oliphant J. y Buitrago D. (2017). “Trump amenaza a Venezuela con intervención militar, Caracas dice que es un 'acto de locura", en Reuters. Disponible en: https://ta.reuters.com/article/topNews/idLTAKBN1AR2H6-OUSLT (Consultado el 9 de mayo de 2018).

Oliva, A. (2018). "Venezuela, más allá de todo", en Nueva Sociedad. Disponible en: http://nuso.org/articulo/venezuela-mas-alla/ (Consultado el 20 de junio de 2018). 
Pence, M. (2017). "Remarks by the Vice President and President Bachelet of Chile in a Joint Press Conference", en The White House. Disponible en: https:// www.whitehouse.gov/briefings-statements/remarks-vice-president-president-bachelet-chile-joint-press-conference/ (Consultado el 17 de mayo de 2018).

Precio del petróleo. Datosmacro.com [gráfico]. (2018). Disponible en: https://www. datosmacro.com/materias-primas/opec (Consultado el 20 de junio de 2018).

Propuesta del Candidato de la Patria Comandante Hugo Chávez para la gestión bolivariana socialista 2013-2019, Partido Socialista Unido de Venezuela [Programa de la Patria]. (2012).

Report of Donations Accepted. Comisión Electoral Federal de EE. UU. (2017). Disponible en: http://docquery.fec.gov/pdf/286/201704180300150286/201704180 300150286.pdf (Consultado el 30 de agosto de 2018).

Romero, M. (2015). "Los desaciertos diplomáticos en el Caribe de Venezuela con Guyana”, en Panam Post. Disponible en: https:/es.panampost.com/mariateresa-romero/2015/08/20/1os-desaciertos-diplomaticos-en-el-caribe-devenezuela-con-guyana/?cn-reloaded $=1$ (Consultado el 29 de mayo de 2018).

Romero, M. (2017). "Petrocaribe, la diplomacia chavista del petróleo, camino a desaparecer", en Panam Post. Disponible en: https://es.panampost.com/mariateresa-romero/2017/11/02/diplomacia-chavista-del-petroleo/?cn-reloaded=1 (Consultado el 10 de octubre de 2018).

Segundo Plan Socialista de Desarrollo Económico y Social de la Nación 20132019, República Bolivariana de Venezuela [Ley]. (2013).

Smilde, D. (2018). “Estados Unidos debe atacar a Venezuela?”, en The New York Times. Disponible en: https://www.nytimes.com/es/2018/01/15/estados-unidos-atacar-venezuela-opinion-smilde/ (Consultado el 20 de junio de 2018).

Solidaridad y respaldo a la institucionalidad democrática, al diálogo y a la paz en la República Bolivariana de Venezuela, Consejo Permanente, OEA [Declaración 51 (1957/14)]. (2014). Disponible en: http://www.oas.org/consejo/sp/ resoluciones/dec51.asp (Consultado el 26 de junio de 2018).

Stargardter, G. (2017). "México analiza remplazar petróleo de Petrocaribe si cae gobierno en Venezuela: fuentes", en Reuters. Disponible en: https://ta.reuters.com/article/domesticNews/idLTAKCN1B31XW-OUSLD (Consultado el 10 de octubre de 2018).

Sutherland, M. (2017). "Venezuela sin fondo... y sin alternativas", en Nueva Sociedad. Disponible en: http://nuso.org/articulo/venezuela-sin-fondo-y-sinalternativas/ (Consultado el 14 de junio de 2018). 
Trump, D., @realDonaldTrump [Tweet de la cuenta verificada de Trump], (2017a). Disponible en: https://twitter.com/realDonaldTrump/status/832016501657968640 (Consultado el 14 de junio de 2018)

Trump, D., (2017b) "Presidential Proclamation Enhancing Vetting Capabilities and Processes for Detecting Attempted Entry in to the United States by Terrorists or Other Public-Safety Threats", en The White House. Disponible en: https://www.whitehouse.gov/presidential-actions/presidential-proclamationenhancing-vetting-capabilities-processes-detecting-attempted-entry-unitedstates-terrorists-public-safety-threats/ (Consultado el 13 de junio de 2018)

\section{Apéndice}

\begin{tabular}{|c|c|c|}
\hline & Plano interno & Plano externo \\
\hline \multirow[t]{4}{*}{2013} & & \\
\hline & Mar- Fallecimiento de Chávez & \\
\hline & $\begin{array}{l}\text { Abr- Maduro se convierte en } \\
\text { presidente }\end{array}$ & \\
\hline & Dic- Sanción Ley de la Patria & \\
\hline \multirow[t]{4}{*}{2014} & Ene/feb- Oleada de protestas & \\
\hline & & $\begin{array}{l}\text { Mar- Consejo Permanente OEA } \\
\text { declaración /UNASUR crea } \\
\text { comisión de cancilleres para el } \\
\text { diálogo }\end{array}$ \\
\hline & $\begin{array}{l}\text { Abr- Conferencia Nacional por } \\
\text { la Paz (Cancilleres UNASUR + } \\
\text { Vaticano) }\end{array}$ & \\
\hline & $\begin{array}{l}\text { May-Clausura de la Conferencia } \\
\text { Nacional por la Paz }\end{array}$ & $\begin{array}{l}\text { May- (EE. UU.) Sanción Ley } \\
\text { Derechos Humanos y Protección de } \\
\text { la Democracia en Venezuela }\end{array}$ \\
\hline \multirow[t]{3}{*}{2015} & & $\begin{array}{l}\text { Mar- Orden Ejecutiva Obama; } \\
\text { Nueva delegación Cancilleres } \\
\text { UNASUR }\end{array}$ \\
\hline & & $\begin{array}{l}\text { May- Luis Almagro asume como } \\
\text { Secr. Gral. OEA }\end{array}$ \\
\hline & Dic- Elecciones parlamentarias & Dic- Asunción Macri (Arg.) \\
\hline
\end{tabular}




\begin{tabular}{|c|c|c|}
\hline \multirow[t]{6}{*}{2016} & $\begin{array}{l}\text { Ene- TSJ declara en desacato a la } \\
\text { AN }\end{array}$ & \\
\hline & & $\begin{array}{l}\text { May- } 1 .^{\circ} \text { Informe Almagro. No } \\
\text { se activa CDI /Propuesta diálogo } \\
\text { UNASUR con expresidentes }\end{array}$ \\
\hline & & Jun- Triunfo Kuczynski (Perú) \\
\hline & & $\begin{array}{l}\text { Ago-Destitución D. Rousseff } \\
\text { (Brasil) }\end{array}$ \\
\hline & $\begin{array}{l}\text { Oct- Inicio diálogo propuesto por } \\
\text { Vaticano }\end{array}$ & $\begin{array}{l}\text { Oct- Inicio diálogo propuesto por } \\
\text { Vaticano }\end{array}$ \\
\hline & & Dic-Triunfo Trump \\
\hline \multirow[t]{8}{*}{2017} & Feb- Fin diálogo & Feb- Fin diálogo \\
\hline & Mar-TSJ asume funciones AN & Mar-No se activa CDI \\
\hline & $\begin{array}{l}\text { Abr-TJS devuelve funciones. AN } \\
\text { sigue en desacato }\end{array}$ & $\begin{array}{l}\text { Abr-Triunfo Lenin Moreno } \\
\text { (Ecuador); } 2 .^{\circ} \text { Informe Almagro; } \\
\text { Venezuela decide abandonar OEA }\end{array}$ \\
\hline & & May-Encuentro Cancilleres OEA \\
\hline & $\begin{array}{l}\text { Jun- Convocatoria Asamblea } \\
\text { Constituyente }\end{array}$ & \\
\hline & $\begin{array}{l}\text { Jul- Consulta popular de la } \\
\text { oposición contra la Constituyente / } \\
\text { Elecciones para la Constituyente }\end{array}$ & \\
\hline & Ago- Se instala la Constituyente & $\begin{array}{l}\text { Ago-Venezuela suspendida de } \\
\text { MERCOSUR; se crea grupo de } \\
\text { Lima }\end{array}$ \\
\hline & Dic-Elecciones municipales & $\begin{array}{l}\text { Dic- Venezuela incluida en la } \\
\text { Nueva Estrategia de Seg. Nac. } \\
\text { EE. UU.; Triunfo Piñera (Chile) }\end{array}$ \\
\hline
\end{tabular}

